\title{
O efeito da eletroestimulação transcutânea na espastici- dade pós acidente vascular cerebral
}

\author{
Effect of transcutaneous electrical stimulation on spasticity after stroke
}

\author{
José Eduardo Pompeu', Sandra Maria Alvarenga Anti Pompeu', Fernanda Iotti \\ Fernandes 3 , Gabriela Alves de Castro ${ }^{3}$, Paulo Roberto Garcia Lucarelí, Thiago \\ Yukio Fukuda
}

\section{RESUMO}

Objetivo. Verificar o efeito da TENS modo burst (TENSb) sobre a espasticidade e a mobilidade de pacientes com hemiparesia após acidente vascular cerebral (AVC). Método. Foram selecionados nove indivíduos (6 homens) com média de idade de 61,3 $\pm 9,3$ anos, hemiparéticos crônicos espásticos. A espasticidade do músculo quadríceps foi avaliada por meio da escala de Ashworth Modificada, pelo teste do pêndulo e seu respectivo índice de relaxamento corrigido (IRC), e pelo teste de Duncan-Ely. A mobilidade foi avaliada pelo teste Time Up and Go (TUG). Foram realizadas quatro aplicaçóes de 30 minutos de TENSb (frequência de $100 \mathrm{~Hz}$ e duração de pulso de $120 \mu \mathrm{s}$ ) em dias consecutivos. Foram feitas avaliaçóes antes e depois das quatro aplicaçôes e após 72 horas da última sessão. Resultados. Houve aumento do IRC após as aplicaçóes de $0,4 \pm 0,2$ para $0,9 \pm 0,3(\mathrm{p}<0,05)$, reduçáo da medida do teste de Duncan-Ely de $10,8 \pm 3,1$ para $7,7 \pm 2,7$ ( $p<0,05$ ) e diminuição de $2,0 \pm 0,6$ para $1,0 \pm 0,4$ na Escala de Ashworth Modificada. Também houve redução no tempo de execução do

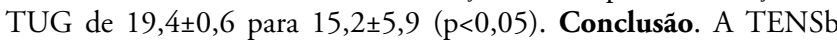
promoveu reduçáo da espasticidade do músculo quadríceps após a aplicação e após 72 horas e promoveu melhora na mobilidade de pacientes pós AVC.

Unitermos. Espasticidade Muscular, Estimulação Elétrica Nervosa Transcutânea, Acidente Vascular Cerebral

Citação. Pompeu JE, Pompeu SMAA, Fernandes FI, Castro GA, Lucareli PRG, Fukuda TY. O efeito da eletroestimulação transcutânea na espasticidade pós acidente vascular cerebral.

\begin{abstract}
Objective. To investigate the effect of burst mode TENS (TENSb) on spasticity and mobility in patients with hemiparesis after stroke. Method. We selected nine subjects (6 males) with a mean age of $61.3 \pm 9.3$ years, who presented chronic spastic hemiparesis. The spasticity of the quadriceps muscle was assessed by the Modified Ashworth Scale, the pendulum test and its corrected relaxation index (CRI), and the Duncan-Ely test (DET). Mobility was assessed by the Time Up and Go (TUG). The application of TENSb (frequency of $100 \mathrm{~Hz}$ and pulse duration of $120 \mu \mathrm{s}$ ) lasted 30 minutes per session for four consecutive days. Assessments were performed before and after four TENSb applications and after 72 hours of the end of the intervention. Results. There was a increase of the CRI from $0.4 \pm 0.2$ to $0.9 \pm 0.3$ ( $p<0.05$ ), decrease of the DET from $10.8 \pm 3.1$ to $7.7 \pm 2.7(\mathrm{p}<0.05)$, and decrease

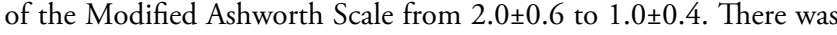
decrease in the time of performance of the TUG from $19.4 \pm 0.6$ to $15.2 \pm 5.9(\mathrm{p}<0.05)$. Conclusion. The TENSb decreased quadriceps muscle spasticity after application and after 72 hours and improved mobility in patients following stroke.
\end{abstract}

Keywords. Muscle Spasticity, Transcutaneous Electrical Nerve Stimulation, Stroke

Citation. Pompeu JE, Pompeu SMAA, Fernandes FI, Castro GA, Lucareli PRG, Fukuda TY. Effect of transcutaneous electrical stimulation on spasticity after stroke. 


\section{INTRODUÇÃO}

O acidente vascular encefálico (AVC) é causado pela alteração do fluxo sanguíneo do encéfalo devido a fenômenos embólicos, trombóticos ou hemodinâmicos, bem como pela hemorragia ocasionada por rupturas de aneurismas ou má formaçóes arteriovenosas ${ }^{1}$. A sequela motora mais comum do AVC é a hemiparesia espástica, caracterizada pela presença de hipertonia elástica, hiperreflexia, sinal de Babinski positivo e clônus, sinais conhecidos como síndrome do neurônio motor superior ${ }^{2}$. A espasticidade do paciente com hemiparesia compromete predominantemente os músculos antigravitacionais causando a manutençáo da postura flexora do membro superior e extensora de membro inferior acometido, associada a sinergias estereotipadas dos movimentos ${ }^{3}$ que podem comprometer o desempenho de atividades funcionais como a marcha e as trocas posturais ${ }^{4}$. Várias abordagens farmacológicas ${ }^{5}$ e não farmacológicas ${ }^{6}$ têm sido realizadas no intuito de controlar a espasticidade nestes pacientes, entre elas a estimulação elétrica nervosa transcutânea (TENS $)^{7}$. Estudos demonstraram que o uso da TENS no tratamento da espasticidade pode auxiliar na restauração de movimentos funcionais, pela supressão da anormalidade do tônus e da atividade do reflexo de estiramento fásico $^{7-11}$. Entretanto, os resultados destes estudos são controversos devido a uma extensa variação de parâmetros de estimulação e duração da intervenção. Estudos sugerem que as contraçóes musculares regulares poderiam reduzir a rigidez articular. No entanto, os efeitos da aplicação da TENS no modo burst sobre a espasticidade ainda são pouco investigados ${ }^{12-14}$. Um estudo analisou o efeito da TENS sobre a espasticidade de indivíduos hemiparéticos. ATENS no modo burst foi aplicada em quatro indivíduos por 30 minutos durante nove dias consecutivos $(100 \mathrm{~Hz}$, largura de pulso de $120 \mu$ s) sobre o músculo quadríceps do membro acometido. A avaliaçáo da espasticidade do músculo quadríceps foi realizada através do teste do pêndulo. Foi observada redução significativa da espasticidade imediatamente após o término da quinta aplicação, mas os efeitos não permaneceram a longo prazo. No entanto, autores não avaliaram a repercussão funcional da redução da espasticidade promovida pela TENS ${ }^{14}$.

Assim, o objetivo desse estudo foi verificar os efei- tos da TENS no modo burst na espasticidade do músculo quadríceps e na mobilidade de pacientes hemiparéticos crônicos pós-AVC. Como desfecho primário da espasticidade foi considerado o Índice de Relaxamento Corrigido (IRC) derivado do teste do pêndulo. Os desfechos secundários foram: (1) a medida do teste de Duncan-Ely; (2) a pontuação da Escala de Ashworth Modificada e (3) o tempo de execução do Time Up and Go (TUG). Foi hipotetizado que a TENS modo burst pode promover redução da espasticidade com repercussão positiva sobre a mobilidade dos pacientes pós AVC.

\section{MÉTODO}

\section{Amostra}

A coleta de dados foi realizada na Clínica Escola da Universidade Paulista, e na Associação Pelos Direitos da Pessoa Deficiente, ambas localizadas em São Paulo, Brasil. Os critérios de elegibilidade foram: presença de um quadro clínico de hemiparesia; tempo de AVC superior a um ano; idade entre 45 e 80 anos; presença de espasticidade no músculo quadríceps $\geq 1+$ na Escala de Ashworth Modificada e independência para a marcha. Foram excluídos os indivíduos que utilizavam drogas anti-espásticas via oral ou intramuscular, que apresentavam marcapasso cardíaco, história pregressa de alteraçóes como ataques epilépticos, tumores, ossificação heterotópica, comprometimento cognitivo grave e deficiência auditiva ou a não concordância com o Termo de Consentimento Livre e Esclarecido. Este estudo foi aprovado pelo Comitê de Ética em Pesquisa envolvendo seres humanos do Centro Universitário São Camilo, no 057/07.

\section{Procedimento}

Os indivíduos do estudo receberam a TENS burst (Endophasis NMS 0501-KLD Biosistemas ${ }^{\oplus}$, Amparo-SP, BR) com frequência de $100 \mathrm{~Hz}$, duração de pulso de $120 \mu$ s e nível de intensidade em nível sensorial de acordo com a tolerância do indivíduo, por 30 minutos, durante quatro dias consecutivos.

Foram utilizados seis eletrodos auto-adesivos de dimensóes $5 \times 5 \mathrm{~cm}$, que foram distribuídos na região do músculo quadríceps segundo os critérios de posicionamento do Surface Electromyography for the Non-Invasive Assessment of Muscles-SENIAM ${ }^{15}$, sobre os músculos reto 
femoral, vasto medial e vasto lateral.

Os indivíduos foram avaliados antes e depois das aplicações e após 72 horas da última aplicação por um avaliador cego em relaçáo aos objetivos do estudo e ao tipo de intervenção que os participantes iriam receber. A avaliação da espasticidade foi realizada por meio da Escala Modificada de Ashworth16, teste do Pêndulo ${ }^{17,18}$ e o teste Duncan-Ely ${ }^{19}$. A mobilidade foi avaliada por meio do teste Time Up and Go (TUG) ${ }^{20}$.

\section{Teste do Pêndulo}

O teste de pêndulo foi utilizado para determinar o tônus do músculo quadríceps ${ }^{17}$. Os indivíduos foram posicionados confortavelmente em decúbito dorsal elevado com o apoio de uma cunha na região das costas que permitia uma angulação de 45 graus na articulação do quadril, para evitar a influência de um possível encurtamento do músculo iliopsoas. Suas pernas permaneceram pendentes sobre a borda da maca, de maneira que a flexão da perna testada ocorresse livremente. $\mathrm{O}$ membro inferior do lado náo acometido foi posicionado sobre um apoio e mantido com 90 graus de flexão da coxa e da perna e o membro a ser avaliado foi posicionado com máxima extensão da perna, sendo sustentado pelo avaliador na região dos maléolos.

O indivíduo foi instruindo a relaxar durante a execução do teste e de modo inesperado, o avaliador liberava o membro, deixando a perna cair, de maneira que esta oscilava livremente sob a ação da gravidade. $\mathrm{O}$ peso da perna promovia um deslocamento no sentido da flexão, levando ao estiramento brusco do músculo quadríceps.

Antes da execução do teste do pêndulo foram fixados quatro marcadores na pele dos indivíduos, sensíveis a raios de infravermelho, na região do trocânter maior do fêmur, espinha ilíaca ântero-superior, cabeça da fíbula e no maléolo lateral do membro inferior acometido (Figura 1). Assim, o teste foi filmado por uma hand cam da marca Sony DV TRV120, com frequência de aquisição de 30 $\mathrm{Hz}$, sobre um tripé que estava posicionado a $1,60 \mathrm{~m}$ de distância do centro da maca e $0,85 \mathrm{~cm}$ de altura em ambiente escuro com um emissor de raios infravermelhos.

Posteriormente, o teste foi analisado pelo programa Maxtraq ${ }^{\oplus}$ (Innovision Systems, Columbiaville, MI, USA) que apresentava a variação do ângulo de flexão da coxa, da perna e do pé durante as oscilaçóes sofridas pela perna pendente.

O registro angular foi utilizado para o cálculo do índice de relaxamento corrigido (IRC), que se correlaciona com o grau de espasticidade. A Figura 2 mostra as oscilaçóes da perna do indivíduo no tempo apresentando os deslocamentos angulares da posição inicial até a posição de repouso final, sendo que indivíduos com espasticidade apresentam um número menor de oscilações. $\mathrm{Na}$ figura 2 , o primeiro vale "A1" representa a primeira queda da perna e "A0" que representa a posição final dessa. O cálculo

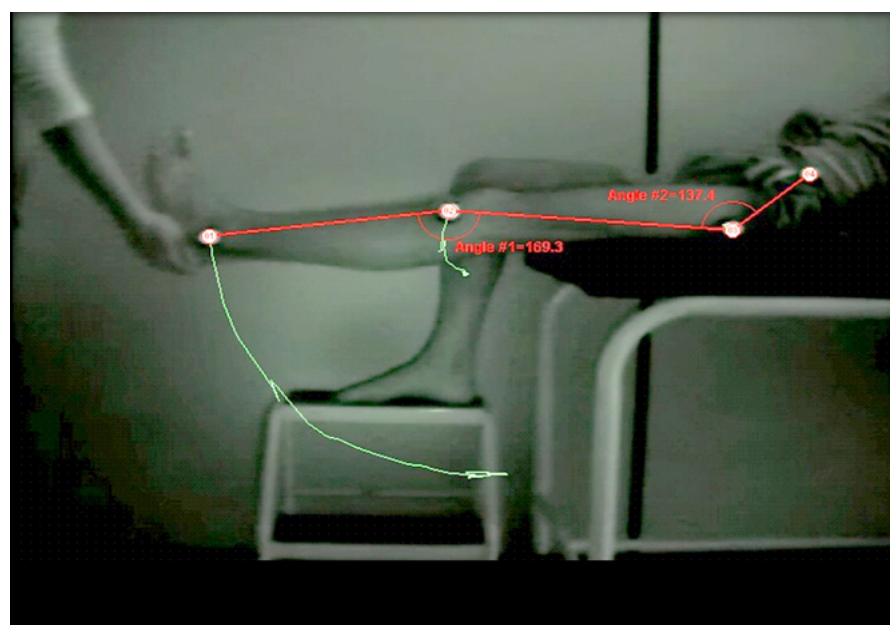

Figura 1. Teste do Pêndulo. O paciente é posicionado em decúbito dorsal com o membro inferior a ser avaliado sustentado pelo avaliador em extensão de $180^{\circ}$. Marcadores reflexivos foram colocados sobre o maléolo lateral, cabeça da fíbula, trocanter maior e espinha iliaca ântero superior. Foto autorizada pelo paciente. 
Figura 2. Representação gráfica do teste do pêndulo, onde A1 representa a variação angular da posição inicial até o valor da máxima angulação atingida na primeira oscilação da perna e AO corresponde à angulação final da perna após o final das oscilaçôes. O Índice de Relaxamento Corrigido18 (IRC) é calculado pela fórmula: $I R C=A 1 / A 0^{*} 1,6$.

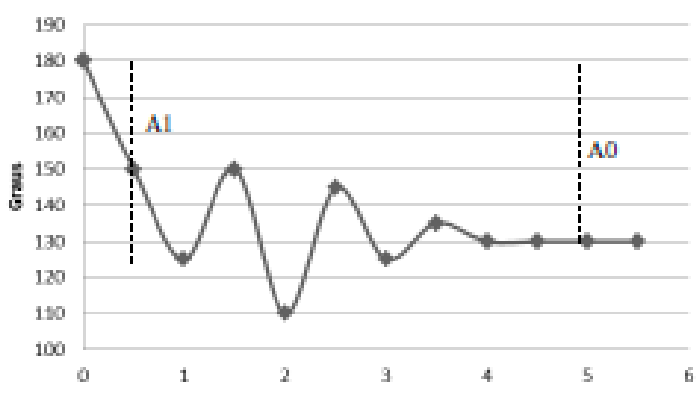

do índice de relaxamento corrigido (IRC) é determinado pela fórmula: IRC=A1/A0*1,6; com 1,6 sendo uma constante de normalidade.

O valor resultante do IRC variando entre valores superiores ou iguais a 1,0 indica um músculo normal e inferiores a 1,0 indicam espasticidade, que é mais grave quanto mais próximo de zero. Após encontrar o IRC das três mediçóes de cada série, determinou-se a média aritmética e o desvio-padrâo dos valores do teste do pêndulo para, posteriormente, estabelecer comparaçóes entre as avaliaçōes.

\section{Teste de Duncan-Ely}

O teste de Duncan-Ely foi realizado com o indivíduo em decúbito ventral na qual examinador realizou uma mobilização passiva rápida da perna do indivíduo provocando o estiramento do músculo reto femoral. Foi mensurada a distância do trocânter maior do fêmur até a maca em que o indivíduo estava ao final da flexão da perna, por meio de uma fita métrica simples graduada em centímetros ${ }^{17}$.

\section{Escala Modificada de Ashworth}

A Escala Modificada de Ashworth ${ }^{13}$ avalia o grau de espasticidade por meio da mobilização passiva realizada no sentido do alongamento muscular. A escala apresenta cinco categorias que variam do tônus normal à rigidez, conforme a resistência muscular contra a movi- mentação passiva do segmento afetado.

\section{Time Up and Go}

A mobilidade dos pacientes foi avaliada pelo Time Up and Go (TUG). Foi solicitado ao indivíduo que levantasse de uma cadeira, caminhasse por três metros em linha reta, contornasse outra cadeira que permanecia oposta à primeira pelo lado de fora, e voltasse à posição inicial. O tempo durante a execução do percurso foi mensurado em segundos por um cronômetro simples durante três tentativas, com um intervalo de descanso igual ao dobro do tempo despendido para o teste anteriormente realizado ${ }^{18}$. Os indivíduos se encontravam descalços e sem auxílio de dispositivos para a marcha.

\section{Análise Estatística}

A análise do IRC foi realizada por meio da ANOVA de medidas repetidas e pelo post hoc teste de Tukey para os valores que atingiram significância estatística. A análise do teste de Duncan-Ely e do TUG foi realizada por meio da ANOVA de medidas repetidas e pelo post hoc de Dunn para os valores que atingiram significância estatística. Foram comparadas as médias dos valores do IRC, do teste de Duncan-Ely e do TUG antes e depois das quatro aplicações e após 72 horas do final da intervenção.

\section{RESULTADOS}

A amostra foi constituída por 9 indivíduos (3 mulheres e 6 homens), média de idade de $61,3 \pm 9,3$ anos, sendo 2 indivíduos com diagnóstico de AVC hemorrágico e 7 com AVC isquêmico (Tabela 1).

Comparando-se os valores do IRC antes e depois de quatro aplicaçóes da TENS, houve aumento com manutenção dos resultados após 72 horas de $0,4 \pm 0,2$ para $0,9 \pm 0,3(p<0,05$; Tabela 2). Portanto, houve redução da espasticidade após quatro aplicaçóes com manutenção dos resultados após 72 horas.

Comparando-se as médias antes e depois de quatro aplicaçóes, houve melhora nos teste de Duncan-Ely de $10,8 \pm 3,1$ para $7,7 \pm 2,7(\mathrm{p}<0,05)$ e no TUG de $19,4 \pm 0,6$ para $15,2 \pm 5,9(\mathrm{p}<0,05)$ com manutenção dos resultados após 72 horas (Tabela 2). 
Tabela 1. Dados demográficos dos pacientes que participaram do presente estudo.

\begin{tabular}{cccccc}
\hline Sujeito & $\begin{array}{c}\text { Idade } \\
\text { (Anos) }\end{array}$ & Sexo & $\begin{array}{c}\text { Tipo } \\
\text { AVC }\end{array}$ & $\begin{array}{c}\text { Tempo } \\
\text { (Anos) }\end{array}$ & EMA \\
\hline $\mathbf{1}$ & 69 & M & I & 3 & $1+$ \\
$\mathbf{2}$ & 53 & M & I & 1,5 & 3 \\
$\mathbf{3}$ & 55 & F & I & 1 & $1+$ \\
$\mathbf{4}$ & 64 & F & I & 3 & $1+$ \\
$\mathbf{5}$ & 46 & M & I & 1 & $1+$ \\
$\mathbf{6}$ & 78 & F & H & 3 & $1+$ \\
7 & 63 & M & I & 1,5 & $1+$ \\
$\mathbf{8}$ & 63 & M & H & 4,5 & 2 \\
$\mathbf{9}$ & 59 & M & I & 1,5 & 3 \\
\hline
\end{tabular}

AVC, acidente vascular encefálico; EMA, escala modificada de Ashworth; M, masculino e $\mathrm{F}$, feminino; $\mathrm{I}$, isquêmico e $\mathrm{H}$, hemorrágico.

Comparando-se as médias da Escala de Ashworth Modificada antes e após as quatro aplicaçóes, houve redução de um ponto em $100 \%$ dos indivíduos, de 2,0 0 0,6 para $1,0 \pm 0,4$, que se manteve após 72 horas.

\section{DISCUSSÂO}

De acordo com os testes realizados antes e após a aplicação de TENS e no follow-up de 72 horas, os resultados do presente estudo nos proporcionaram dois achados principais: (1) a TENS modo burst promoveu efeitos positivos na espasticidade e na mobilidade de pacientes hemiparéticos; (2) estes efeitos permaneceram após 72 horas do final das aplicaçôes, sugerindo sua manutenção em longo prazo.

Há evidências de que a TENS pode promover redução da espasticidade quando aplicada por um período contínuo $^{21}$. Um estudo que avaliou os efeitos da TENS e do baclofeno em indivíduos com lesão medular não observou redução da espasticidade após uma única aplicação de 15 minutos (frequência de $100 \mathrm{~Hz}$ ) sobre o nervo tibial, mas houve efeitos positivos após 15 sessões de aplicação, como, por exemplo, a redução gradativa da Escala de Ashworth Modificada no período de 24 horas após a última aplicação?. Estes resultados estão de acordo com os obtidos em nosso estudo que verificou a reduçáo da espasticidade após quatro aplicaçóes da TENS burst com manutenção do efeito após 72 horas.

Entretanto, outro estudo utilizou a TENS convencional $(100 \mathrm{~Hz}$, durante 20 minutos nos dermátomos L3 e L4) em seis indivíduos com lesão medular incompleta e verificaram melhora da espasticidade, avaliada por meio do teste do pêndulo, imediatamente após o uso da corrente com permanência de seus efeitos por até 2 horas ${ }^{22}$. Achados similares foram verificados no estudo que utilizou a TENS convencional $(100 \mathrm{~Hz}$, uma aplicação de 20 minutos) sobre o nervo sural de indivíduos hemiplégicos e observaram que, em 18 dos 20 indivíduos, houve uma diminuição na resistência muscular durante a movimentação passiva, acompanhada por uma diminuição na atividade eletromiográfica reflexa, que perduraram por 45 minutos após o término da aplicação ${ }^{23}$.

Um ensaio clínico controlado sobre o efeito da TENS modo breve-intenso $(100 \mathrm{~Hz}$, durante 20 minutos em 20 aplicações diárias) em 10 indivíduos com esclerose múltipla constatou redução da espasticidade pela Escala de Ashworth Modificada e pela eletromiografia após quatro semanas de aplicação ${ }^{24}$. Os mesmos efeitos foram observados por um estudo no qual a TENS foi aplicada com frequência de estimulação de $20 \mathrm{~Hz}$, durante 24 sessões de 20 minutos na regiáo do músculo gastrocnêmio de 24 indivíduos com diagnóstico de $\mathrm{AVC}^{25}$. Resultados semelhantes foram encontrados por meio da aplicação da TENS modo acupuntura em 88 indivíduos com diagnóstico de AVC $(100 \mathrm{~Hz}$ em 4 pontos de acupuntura na região de tríceps sural, durante 20 aplicaçóes de 60 minutos) $)^{26}$.

Outros estudos compararam os efeitos da TENS aplicada em alta $(100 \mathrm{~Hz})$ e baixa frequência $(2 \mathrm{~Hz})$ em pontos de acupuntura por um período de 3 meses em pacientes pós $\mathrm{AVC}^{27}$ e após lesão medular ${ }^{28}$. Os autores observaram que apenas a estimulaçâo de alta frequência foi efetiva, sugerindo que a inibição da espasticidade possa

Tabela 2. Resultados obtidos no teste do pêndulo (IRC), Duncan-Ely e TUG, antes e depois das quatro aplicaçōes da TENS burst e após 72 horas.

\begin{tabular}{cccc}
\hline Teste & $\begin{array}{c}\text { Antes das } \\
\text { aplicaçóes }\end{array}$ & $\begin{array}{c}\text { Após a 4a } \\
\text { aplicaçáo }\end{array}$ & $\begin{array}{c}\text { Follow up } \\
\mathbf{7 2} \text { horas }\end{array}$ \\
\hline IRC & $0,4 \pm 0,2^{\mathrm{a}}$ & $0,9 \pm 0,3^{\mathrm{a}}$ & $0,8 \pm 0,3$ \\
TDE (cm) & $10,8 \pm 3,1^{\mathrm{b}}$ & $7,7 \pm 2,7^{\mathrm{b}}$ & $8,5 \pm 2,6$ \\
TUG (s) & $19,4 \pm 0,6^{\mathrm{b}}$ & $15,2 \pm 5,9^{\mathrm{b}}$ & $14,9 \pm 5,6$ \\
\hline
\end{tabular}

Valores em Média $\pm D P$ (desvio padrăo); IRC = índice de relaxamento corrigido; $\mathrm{TDE}=$ teste de Duncan-Ely em centímetros $(\mathrm{cm}) ; \mathrm{TUG}=$ Time Up and Go em segundos (s); $\mathrm{a}=$ ANOVA e pós hoc teste de Tukey, $\mathrm{p}<0,05 ; \mathrm{b}=$ ANOVA e pós hoc teste de Dunn, $\mathrm{p}<0,05$. 
estar relacionada com a liberação de opióides endógenos pelo sistema nervoso central. De modo geral, os estudos verificaram que a TENS de alta frequência $(100 \mathrm{~Hz})$ é mais eficaz na inibição da espasticidade, o que corrobora os resultados do presente estudo que usou a TENS burst, um modo de corrente que também usa alta frequência, porém modulada em "trens de pulso" ou "bursts" que atuam mais especificamente no limiar motor de estimulação.

Após quatro aplicaçóes da TENS houve redução no tempo de desempenho do TUG e redução da espasticidade de acordo com os resultados nos testes do pêndulo, teste de Duncan-Ely e da Escala de Ashworth Modificada, resultados que, em alguns aspectos, corroboram com os achados do estudo de Chen et $\mathrm{al}^{25}$ que verificaram a diminuição no tempo da marcha no teste da caminhada de 10 minutos em indivíduos pós AVC que receberam a TENS $^{25}$. No entanto, houve algumas diferenças metodológicas entre os estudos. Primeiro, o local de estimulação utilizado no presente estudo foi o músculo quadríceps e no estudo de Chen et $\mathrm{al}^{25}$ foi a junção miotendínea do gastrocnêmio. Segundo, além da avaliação da espasticidade por meio da Escala Modificada de Ashowth, os autores utilizaram testes eletrofisiológicos analisando a relação entre as ondas F e M máximas (Fmax/Mmax ratio) e o reflexo H. Terceiro, os parâmetros de estimulação utilizados pelos estudos também diferiram. No estudo de Chen et $\mathrm{al}^{25}$, os autores aplicaram a TENS por 20 minutos, uma vez por dia, seis dias por semana por um mês, enquanto que no presente estudo a TENS foi aplicada em quatro dias consecutivos durante 30 minutos cada aplicação. Finalmente, o presente estudo avaliou a marcha por meio do TUG e o outro estudo analisou o teste de caminhada de 10 minutos. As diferenças nos métodos de aplicação e de avaliação da espasticidade e da marcha dificultam a comparação dos resultados entre os estudos.

A espasticidade traz um impacto negativo na qualidade de vida e na independência funcional de pacientes neurológicos ${ }^{29}$ e, portanto, a melhora da mobilidade observada nos pacientes do presente estudo após a aplicação da TENS pode estar relacionada com a redução da espasticidade promovida pela estimulação. Com isso, nossos resultados demonstraram que a TENS modo burst não somente reduziu a espasticidade como também pode pro- mover melhora na mobilidade. A TENS pode promover a redução da espasticidade pelos mecanismos de facilitação da inibição recorrente das células de Renshaw ${ }^{23}$ ou por diferentes mecanismos fisiológicos como a inibição recíproca do antagonista, a habituação sensorial cutânea, e a diminuição da sensibilidade ao estiramento do fuso muscular através dos seus efeitos sobre o motoneurônio gama $^{20}$. A TENS poderia ainda melhorar o controle do mecanismo inibitório pré-sináptico e ativação das fibras $\mathrm{Ia}^{21}$.

A espasticidade pode ocorrer devido a uma redução da inibição pré-sináptica e sua redução pela TENS poderia ocorrer devido a estimulação deste mecanismo ${ }^{3}$. A inibição pré-sináptica é mediada pelo interneurônio que é ativado tanto por vias descendentes quanto por fontes segmentares e, desta forma, um forte incremento dos impulsos aferentes sobre o interneurônio comum poderia compensar parcialmente a perda sofrida da fonte central ${ }^{3}$. Outros estudos sugerem que a TENS pode promover diminuição da co-contração de antagonistas espásticos e desinibição dos comandos voluntários descendentes dos motoneurônios alfa ${ }^{23,24}$.

O presente estudo apresenta algumas limitaçóes como o número reduzido de sujeitos e a falta de um grupo placebo-controle. No entanto, os resultados apresentados sugerem que o uso da TENS burst pode ser um coadjuvante no tratamento da espasticidade de pacientes com sequela de AVC e contribuir para a melhora da mobilidade

\section{CONCLUSÃO}

A TENS burst promoveu redução da espasticidade do músculo quadríceps e contribuiu para melhora na mobilidade dos indivíduos hemiparéticos espásticos. Foram observados efeitos positivos após quatro aplicaçóes que permaneceram após 72 horas sem a aplicação da corrente.

\section{AGRADECIMENTOS}

Os autores agradecem a Universidade Paulista e a Associação Pelos Direitos da Pessoa Deficiente de São Paulo. 


\section{REFERÊNCIAS}

1.Shaller CA, Jacques S, Shelden CH. The pathophysiology of stroke: a review with molecular considerations. Surg Neurol 1980;14:433-43.

2.Wissel J, Manack A, Brainin M. Toward an epidemiology of poststroke spasticity. Neurology 2013;80(Suppl 2):S13-9. http://dx.doi.org/10.1212/ WNL.0b013e3182762448

3.Ward AB. A literature review of the pathophysiology and onset of post-stroke spasticity. Eur J Neurol 2012;19:21-7. http://dx.doi.org/10.1111/j.14681331.2011.03448.x

4.Sunnerhagen KS, Olver J, Francisco GE. Assessing and treating functional impairment in poststroke spasticity. Neurology 2013;80(3 Suppl 2):S35-44. http://dx.doi.org/10.1212/WNL.0b013e3182764aa2

5.Bakheit AM. The pharmacological management of post-stroke muscle spasticity. Drugs Aging 2012;29:941-7. http://dx.doi.org/10.1007/s40266-012$\underline{0034-\mathrm{z}}$

6.Smania N, Picelli A, Munari D, Geroin C, Ianes P, Waldner A, et al. Rehabilitation procedures in the management of spasticity. Eur J Phys Rehabil Med 2010;46:423-38.

7.Shaygannejad V, Janghorbani M, Vaezi A, Haghighi S, Golabchi K, Heshmatipour M. Comparison of the effect of baclofen and transcutaneous electrical nerve stimulation for the treatment of spasticity in multiple sclerosis. Neurol Res 2013;35:636-41. http://dx.doi.org/10.1179/1743132813Y.0000000200 8.Amatya B, Khan F, La Mantia L, Demetrios M, Wade DT. Non pharmacological interventions for spasticity in multiple sclerosis. Cochrane Database Syst Rev 2013;2:CD009974. http://dx.doi.org/10.1002/14651858.CD009974. pub2

9.Aydin G, Tomruk S, Keleş I, Demir SO, Orkun S. Transcutaneous electrical nerve stimulation versus baclofen in spasticity: clinical and electrophysiologic comparison. Am J Phys Med Rehabil 2005;84:584-92. http://dx.doi. org/10.1097/01.phm.0000171173.86312.69

10. Cho HY, In TS, Cho KH, Song CH. A single trial of transcutaneous electrical nerve stimulation (TENS) improves spasticity and balance in patients with chronic stroke. Tohoku J Exp Med 2013;229:187-93. http://dx.doi. org/10.1620/tjem.229.187

11.Ping Ho Chung B, Kam Kwan Cheng B. Immediate effect of transcutaneous electrical nerve stimulation on spasticity in patients with spinal cord injury. Clin Rehabil 2010;24:202-10. http://dx.doi.org/10.1177/0269215509343235

12.Lundeberg T. Electrical stimulation for the relief of pain. Physiother 1984;70:98-100.

13.Gersh MR, Wolf SL. Applications of transcutaneous electrical nerve stimulation in the management of patients with pain. State-of-the-art update. Phys Ther 1985;65:314-36.

14.Silva KG, Pompeu JE, Fukuda TY. Efeito da estimulação elétrica transcutânea do nervo sobre a espasticidade de indivíduos hemiparéticos. Med Reabil 2009;28:55-8.

15.Hermens HJ, Freriks B, Disselhorst-Klug C, Rau G. Development of recommendations for SEMG sensors and sensor placement procedures. J Electromyogr Kinesiol 2000;10:361-74. http://dx.doi.org/10.1016/S1050$\underline{6411(00) 00027-4}$
16.Ansari NN, Naghdi S, Mashayekhi M, Hasson S, Fakhari Z, Jalaie S. Intra-rater reliability of the Modified Modified Ashworth Scale (MMAS) in the assessment of upper-limb muscle spasticity. NeuroRehab 2012;31:215-22. http://dx.doi.org/10.3233/NRE-2012-0791

17.Fowler EG, Nwigwe AI, Ho TW. Sensitivity of the pendulum test for assessing spasticity in persons with cerebral palsy. Dev Med Child Neurol 2000;42:182-9. http://dx.doi.org/10.1111/j.1469-8749.2000.tb00067.x

18.Jamshidi M, Smith AW. Clinical measurement of spasticity using the pendulum test: comparison of electrogoniometric and videotape analyses. Arch Phys Med Rehabil 1996;77:1129-32. http://dx.doi.org/10.1016/S00039993(96)90134-3

19.Marks MC, Alexander J, Sutherland DH, Chambers HG. Clinical utility of the Duncan-Ely test for rectus femoris dysfunction during the swing phase of gait. Dev Med Child Neurol 2003;45:763-8. http://dx.doi. org/10.1111/j.1469-8749.2003.tb00886.x

20.Yelnik A, Bonan I. Clinical tools for assessing balance disorders. Neurophysiol Clin 2008;38:439-45. http://dx.doi.org/10.1016/j.neucli.2008.09.008

21.Levin MF, Hui-Chan CW. Relief of hemiparetic spasticity by TENS is associated with improvement in reflex and voluntary motor functions. Electroencephalogr Clin Neurophysiol 1992;85:131-42. http://dx.doi.org/10.1016/0168-5597(92)90079-Q

22.Bajd T, Gregoric M, Vodovnik L, Benko H. Electrical stimulation in treating spasticity resulting from spinal cord injury. Arch Phys Med Rehabil 1985;66:515-7.

23.Robbins SM, Houghton PE, Woodbury MG, Brown JL. The therapeutic effect of functional and transcutaneous electric stimulation on improving gait speed in stroke patients: a meta-analysis. Arch Phys Med Rehabil 2006;87:8539. http://dx.doi.org/10.1016/j.apmr.2006.02.026

24.Armutlu K, Meriç A, Kirdi N, Yakut E, Karabudak R. The effect of transcutaneous electrical nerve stimulation on spasticity in multiple sclerosis patients: a pilot study. Neurorehabil Neural Repair 2003;17:79-82. http://dx.doi. org/10.1177/0888439003017002001

25.Chen SC, Chen YL, Chen CJ, Lai CH, Chiang WH, Chen WL. Effects of surface electrical stimulation on the muscle-tendon junction of spastic gastroc nemius in stroke patients. Disabil Rehabil 2005;27:105-10. http://dx.doi. org/10.1080/09638280400009022

26.Ng SS, Hui-Chan CW. Transcutaneous electrical nerve stimulation combined with task-related training improves lower limb functions in subjects with chronic stroke. Stroke 2007;38:2953-9. http://dx.doi.org/10.1161/STROKEAHA.107.490318

27.Yu Y. [Transcutaneous electric stimulation at acupoints in the treatment of spinal spasticity: effects and mechanism]. Zhonghua Yi Xue Za Zhi 1993;73:593-5.

28.Han JS, Chen XH, Yuan Y, Yan SC. Transcutaneous electrical nerve stimulation for treatment of spinal spasticity. Chin Med J (Engl). 1994;107:6-11.

29.Schinwelski M, Sławek J. Prevalence of spasticity following stroke and its impact on quality of life with emphasis on disability in activities of daily living. Systematic review. Neurol Neurochir Pol 2010;44:404-11. http://dx.doi. org/10.1016/S0028-3843(14)60300-5 